\title{
Brownian diode: Molecular motor based on a semi-permeable Brownian particle with internal potential drop
}

\author{
A.V. Plyukhin \\ Department of Mathematics, Saint Anselm College, Manchester, New Hampshire 03102, USA
}

\begin{abstract}
A model of an autonomous isothermal Brownian motor with an internal propulsion mechanism is considered. The motor is a Brownian particle which is semi-transparent for molecules of surrounding ideal gas. Molecular passage through the particle is controlled by a potential similar to that in the transition rate theory, i.e. characterized by two stationary states with a finite energy difference separated by a potential barrier. The internal potential drop maintains the diode-like asymmetry of molecular fluxes through the particle, which results in the particle's stationary drift.
\end{abstract}

Keywords: Molecular motors, active transport, Brownian motion

\section{Introduction}

Most natural (biological) molecular motors and many artificial molecular machines are powered by chemical reactions. Particular ways to convert chemical energy to mechanical work vary considerably for different classes of motors. A number of prototypical models were suggested which are usually rather involved and require application of numerical methods [1, 2, 3, 3, , 5]. The purpose of this paper is to introduce a new simple model of autonomous Brownian motor in which the complexity of involved chemical processes is hidden in a single parameter of an internal potential drop, not dissimilar to how an electric battery is characterized by its voltage.

Our motor is essentially a Brownian particle permeable for molecules of the surrounding thermal bath, which can flow in and out the particle. As a physical realization of permeable Brownian particles one can mention for instance micro-gels [6], and lipid vesicles like liposomes [7]. When a molecule is inside the particle, it experiences a potential $U(x)$ similar to that in the transition-state theory. Calculations are particularly simple for a linear piecewise potential $U(x)$ depicted in Fig. 1. It is characterized, first, by the presence of a barrier which prevents the crossing of the particle by low-energy molecules, and, secondly, by a nonzero potential difference $\Delta U$ between the two sides from the barrier. As discussed below, the internal propulsion force is generated by the potential drop $\Delta U$ which, similar to diode devices, results in asymmetry of molecular fluxes through the particle and a nonzero average momentum it receives in unit time.

In the non-operational regime, when the average molecular flux through the particle is zero, a potential profile like that in Fig. 1 can be formed naturally, for instance as a result of asymmetric ion distribution across the particle. The formation of nonzero potential difference between the two sides of asymmetric lipid bilayers was discussed in [8]. However, it is evident that an external energy source is necessary to maintain an asymmetric charge distribution and internal potential drop in the operational regime of nonzero flux across the particle (otherwise the system would violate the second law of thermodynamics). The function of a "battery" sustaining a stationary potential drop $\Delta U$ can be performed by particle-bound ATP-or light driven ion pumps, capable of moving ions against potential and/or concentration gradients (active transport) [9, 10]. Certain proteins, which act as ion pumps in living cells, are able to retain their function when transplanted into artificial membrane structures [11]. This appears to provide a technologically feasible way to synthesize permeable Brownian objects with an internal potential drop. 


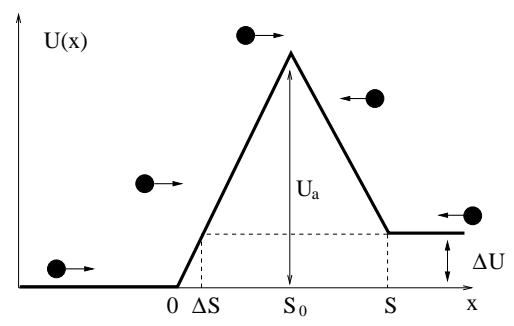

Figure 1: Potential energy $U(x)$ of molecules interacting with the permeable Brownian particle of size $S$. Coordinates $x=0$ and $x=S$ correspond to the left and right edges of the particle. Molecules (black circles) "see" the barrier of height $U_{a}$ when coming from the left, and $U_{a}-\Delta U$ when coming from the right. For $\Delta U>0$, the particle drifts to the right.

The model presented here is phenomenological in the sense that specific mechanisms of active transport involved to sustain the potential drop $\Delta U$ are not identified. Of course, such an approach does not allow for the determination of the motor's efficiency [12], but is sufficient to evaluate transport characteristics of the motor as a function of $\Delta U$, which is a goal of this paper.

\section{Model}

We consider a permeable Brownian particle of size $S$ and mass $M$ immersed in a thermal bath of ideal gas molecules of mass $m \ll M$. The particle and molecules are constrained to move in one dimension, molecular distribution far from the particle is homogeneous with concentration $n$, and the velocity distribution of molecules is Maxwellian

$$
f_{M}(v)=\frac{1}{v_{T} \sqrt{2 \pi}} \exp \left\{-\frac{1}{2}\left(\frac{v}{v_{T}}\right)^{2}\right\},
$$

where $v_{T}=1 / \sqrt{m \beta}$ and $\beta=1 / k_{B} T$. Molecules interact with the particle with a piecewise linear potential $U(x)$ depicted in Fig. 1. The direct interaction of molecules to each other is assumed to be negligible. Suppose the particle's interior corresponds to the interval $0<x<S$, with $x=S_{0}$ being the position of the barrier's maximum. When inside the particle, a molecule experiences a constant force $f=|d U / d x|$ directed to the left on the left slope $0<x<S_{0}$, and to the right on the right slope $S_{0}<x<S$. The right arm of the potential is shorter than the left one by $\Delta S$, which results in the difference of baseline potential values (potential drop) $\Delta U=f \Delta S$.

The idealized model potential like that in Fig.1 can be formed by two electric double layers with opposite polarities, infinitely extended in the $y$ and $z$ directions. In a realistic case of the particle of finite dimensions, one has to take into account a nonzero field outside the particle and, in general, a discontinuity of the potential at the particle boundary [13], but these would not change the problem qualitatively.

It is intuitively clear that for given geometry the average force exerted on the particle by molecules coming from the left exceeds the force from molecules coming from the right, and thus the particle would drift to the right. Our aim is to find the stationary drift velocity of the particle $\langle V\rangle$. (A more simple model with a monotonic step-like potential $U(x)$ shows a nonzero drift, but no friction, and therefore, in our opinion, is of less interest.)

In lowest order in the mass ratio parameter $m / M$, which is sufficient for our purposes, one can justify the standard Langevin equation for the particle's velocity $V$,

$$
M \frac{d V(t)}{d t}=-\gamma V(t)+F(t)
$$

where however the fluctuating force $F(t)$ is not zero-centered, so that the average stationary drift velocity is

$$
\langle V\rangle=\frac{\langle F\rangle}{\gamma} .
$$


Even for the simple model outlined above, the analytic evaluation of $\langle F\rangle$ and $\gamma$ is rather involved except when the potential drop is small and the barrier is high,

$$
\beta \Delta U \ll 1, \quad \beta U_{a} \gtrsim 1
$$

The first condition allows one to evaluate the average fluctuating force in a linear approximation in $\Delta U$, in which case the drift velocity can be written as

$$
\langle V\rangle=\sigma \Delta U
$$

where the parameter $\sigma$ can be called the mobility. The second condition, $\beta U_{a} \gtrsim 1$, implies that the friction coefficient $\gamma$ can be calculated in the limit of an infinitely high barrier, when the fluctuating force $F(t)$ is zero-centered, and the standard fluctuation-dissipation relation holds

$$
\gamma=\beta \int_{0}^{\infty} d t\langle F(0) F(t)\rangle .
$$

From a practical point of view the conditions (44) are of less interest since the corresponding mobility $\sigma$ is very small. Yet in what follows we evaluate $\sigma$ assuming for the sake of simplicity that conditions (4) are satisfied. One naturally expects that in this case the temperature dependence is given by an Arrhenius law $\sigma=\sigma_{0} \exp \left(-\beta U_{a}\right)$, so that our major interest will be in the pre-exponential factor $\sigma_{0}$.

\section{Impermeable particle}

In this section, we evaluate the correlation function of the Langevin fluctuating force $F(t)$ and, using the fluctuation-dissipation relation (6), the friction coefficient $\gamma$, for the case of a high barrier or low temperature, when the molecular barrier crossing is negligible. Clearly, in this case of an impermeable particle the potential drop does not play any physical role and can be set to zero, $\Delta U=0$. Recall that we need $\gamma$ in order to find the drift velocity of the motor with Eq.(3). As will be found below, for the given model

$$
\gamma=4 \sqrt{\frac{2}{\pi}} n m v_{T},
$$

where $n$ is the concentration of bath molecules. Equipped with this result, the reader not interested in derivation details may skip the rest of this section. Yet, since our evaluation is microscopic and exact, it is also a problem of interest of its own. It is also not entirely distracting since in the next section a similar method will be applied to evaluate $\langle F\rangle$ for a permeable particle.

It is convenient to write the fluctuating force as the sum of contributions from molecules coming from the left and right, $F=F_{L}+F_{R}$. For the given case $F$ is zero centered, $\langle F\rangle=0$, and the statistical properties of $F_{L}$ and $F_{R}$ are the same. Then it is sufficient to consider only one, say the "left", contribution, since the correlation function of the total force can be written as

$$
\langle F(0) F(t)\rangle=2\left\langle\left\langle F_{L}(0) F_{L}(t)\right\rangle\right\rangle
$$

where double brackets denote the cumulant $\langle\langle A B\rangle\rangle=\langle A B\rangle-\langle A\rangle\langle B\rangle$.

As known from the microscopic theory of Brownian motion [16], to lowest order in the small mass ratio parameter $m / M$ the fluctuation force $F(t)$ can be evaluated neglecting the particle's motion, i.e. as a pressure on an infinitely heavy particle. Suppose the particle's left edge is fixed at $x=0$, so that $L$-molecules (coming from the left) experience the potential $U(x)$ which equals $f x$ for $x>0$ and zero for $x<0$, see Fig. 2. Each $L$-molecule inside the particle $(x>0)$ gives the same contribution $f$ to the total force on the particle, which therefore can be written as

$$
F_{L}(t)=f \int_{-\infty}^{\infty} d v \int_{0}^{\infty} d x N(x, v, t)
$$



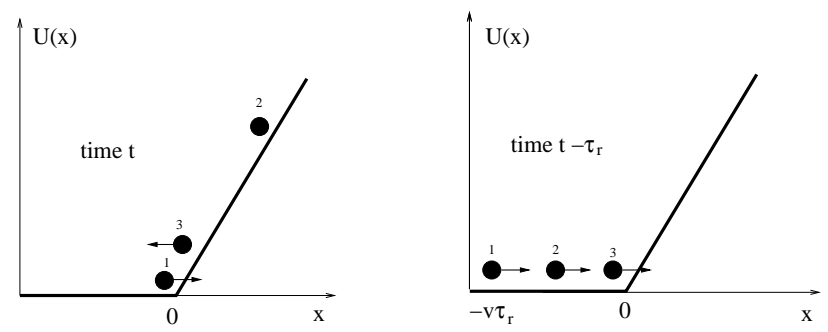

Figure 2: Left figure: a snapshot at time $t$ of three $L$-molecules with the same initial velocity $v$ which are inside the particle $(x>0)$. Arrows show velocity directions. Molecule 1 has just entered the particle, molecule 2 has reached a maximal position, molecule 3 is about to leave the particle's interior. Right figure: a snapshot of the same molecules at an earlier time $t-\tau_{r}$. All three molecules are outside the particle and occupy the interval $-v \tau_{r}<x<0$.

where

$$
N(x, v, t)=\sum_{i} \delta\left(x-x_{i}\right) \delta\left(v-v_{i}\right)
$$

is the microscopic phase density of $L$ molecules.

Let us define the residence time $\tau_{r}(v)$ as the time which an $L$-molecule with initial velocity $v$ spends inside the particle, i.e. in the region $x>0$. Suppose a molecule enters the particle at $t=0$ with the initial velocity $v$. Inside the particle the velocity changes with time as $v(t)=v-(f / m) t$. At the moment when the molecule leaves the particle, its velocity is $-v$. Then the equation $v-(f / m) \tau_{r}=-v$ gives the residence time

$$
\tau_{r}(v)=\frac{2 m v}{f}
$$

For brevity, we shall often suppress the argument $v$ of the function $\tau_{r}(v)$.

In order to evaluate the correlation function of the force $F_{L}(t)$, it is convenient to express it in terms of phase density at an earlier time $t-\tau_{r}$. All molecules, which at time $t$ are inside the particle $x>0$, at time $t-\tau_{r}$ were outside in the region $-v \tau_{r}<x<0$, see Fig. 2. Then, instead of (9), one can write

$$
F_{L}(t)=f \int_{0}^{\infty} d v \int_{-v \tau_{r}}^{0} d x N\left(x, v, t-\tau_{r}\right)
$$

The advantage of this form is that it refers to molecules in the potential free region $x<0$ where the velocities of molecules are constant, so that the following relation holds

$$
N(x, v, t)=N(x-v t, v, 0) \equiv N_{0}(x-v t, v),
$$

where the notation $N_{0}(x, v)$ is introduced for the density at time $t=0$. This relation allows one to reduce temporal correlations to single-time correlation in phase space. For an ideal gas the latter takes the form

$$
\left\langle\left\langle N_{0}(x, v) N_{0}\left(x^{\prime}, v^{\prime}\right)\right\rangle=n f_{M}(v) \delta\left(v-v^{\prime}\right) \delta\left(x-x^{\prime}\right),\right.
$$

where $f_{M}(v)$ is the Maxwellian distribution (1), and $n$ is the density of the gas.

With the above relations, the evaluation of the correlation function for the force $F_{L}(t)$ can be done as follows. From (12), one gets

$$
\left\langle\left\langle F_{L}(t) F_{L}(0)\right\rangle\right\rangle=f^{2} \int_{0}^{\infty} d v \int_{0}^{\infty} d v^{\prime} \int_{-v \tau_{r}}^{0} d x \int_{-v^{\prime} \tau_{r}^{\prime}}^{0} d x^{\prime}\left\langle\left\langle N\left(x, v, t-\tau_{r}\right) N\left(x^{\prime}, v^{\prime},-\tau_{r}^{\prime}\right)\right\rangle\right\rangle,
$$

where $\tau_{r}^{\prime}=\tau_{r}\left(v^{\prime}\right)$. Using (13), we can write the cumulant of the phase density which appears here as

$$
\left\langle\left\langle N_{0}\left(x-t v+\tau_{r} v, v\right) N_{0}\left(x^{\prime}+\tau_{r}^{\prime} v^{\prime}, v^{\prime}\right)\right\rangle\right\rangle .
$$


From (14)-(16), after integration over $v^{\prime}$, one obtains

$$
\left\langle\left\langle F_{L}(t) F_{L}(0)\right\rangle\right\rangle=n f^{2} \int_{0}^{\infty} d v f_{M}(v) \int_{-v \tau_{r}}^{0} d x \int_{-v \tau_{r}}^{0} d x^{\prime} \delta\left(x-t v-x^{\prime}\right) .
$$

The integral of the delta function over $x^{\prime}$ equals one when

$$
-v \tau_{r}<x-t v<0
$$

and zero otherwise. Since Eq.(17) involves only negative $x$, the right side of the condition (18) is satisfied (we assume $t>0$ ), while the left side requires $x>-v\left(\tau_{r}-t\right)$. Then Eq. (17) takes the form

$$
\left\langle\left\langle F_{L}(t) F_{L}(0)\right\rangle\right\rangle=n f^{2} \int_{0}^{\infty} d v f_{M}(v) \int_{-v \tau_{r}}^{0} d x \theta\left(x+v\left(\tau_{r}-t\right)\right)=n f^{2} \int_{0}^{\infty} d v f_{M}(v) v\left(\tau_{r}-t\right) \theta\left(\tau_{r}-t\right),
$$

where $\theta(x)$ is the Heaviside step-function. Recalling expression (11) for the residence time, one obtains

$$
\left\langle\left\langle F_{L}(t) F_{L}(0)\right\rangle\right\rangle=2 n m f \int_{f t / 2 m}^{\infty} d v f_{M}(v)\left(v^{2}-\frac{f t}{2 m} v\right)
$$

which eventually gives

$$
\langle F(t) F(0)\rangle=2\left\langle\left\langle F_{L}(t) F_{L}(0)\right\rangle\right\rangle=2 n m f v_{T}^{2} \operatorname{erfc}\left(\frac{t}{t_{0}}\right),
$$

where $\operatorname{erfc}(x)=1-\operatorname{erf}(x)$ is the complementary error function, and the characteristic time of correlation is $t_{0}=\sqrt{2} \tau_{r}\left(v_{T}\right)=2 \sqrt{2} m v_{T} / f$. Then for the friction coefficient $\gamma$ we obtain the result (7),

$$
\gamma=\beta \int_{0}^{\infty} d t\langle F(t) F(0)\rangle=4 \sqrt{\frac{2}{\pi}} n m v_{T} .
$$

It is interesting to note that this expression, which we found here for a linear potential $U(x)$ (constant repulsive force), coincides with $\gamma$ for a similar model where molecules interact with the particle with a parabolic potential (linear repulsive force) [14]. The same result for $\gamma$ can be obtained from the model with instantaneous (and therefore binary) particle-molecule collisions [15]. For these models correlation functions $\langle F(0) F(t)\rangle$ are different, but the integral (22) is the same.

\section{Permeable particle}

In this section we assume that the potential barrier height is large but finite, $\beta U_{a} \gtrsim 1$, so that hot molecules of the thermal bath can pass through the particle. For a non-zero internal potential drop, $\Delta U \neq 0$, the fluctuating force $F$ in the Langevin equation (2) is not zero-centered, which causes the particle's drift. Our goal is to evaluate $\langle F\rangle$ and the particle's drift velocity $\langle V\rangle=\langle F\rangle / \gamma$ (with $\gamma$ found in the previous section) within a linear approximation, treating $\Delta U$ as a small perturbation.

As in the previous section, we assume that the particle occupies the interval $0<x<S$, and we write the force as the sum of contributions from molecules coming from the left and from the right, $F=F_{L}+F_{R}$. Consider first $F_{L}$. It is convenient to analyze it as the sum of two parts,

$$
F_{L}=F_{L}^{(1)}+F_{L}^{(2)}
$$

The first part $F_{L}^{(1)}$ comes from "slow" molecules with initial velocities (before entering the particle) less than the activation velocity

$$
v_{a}=\sqrt{\frac{2 U_{a}}{m}}
$$



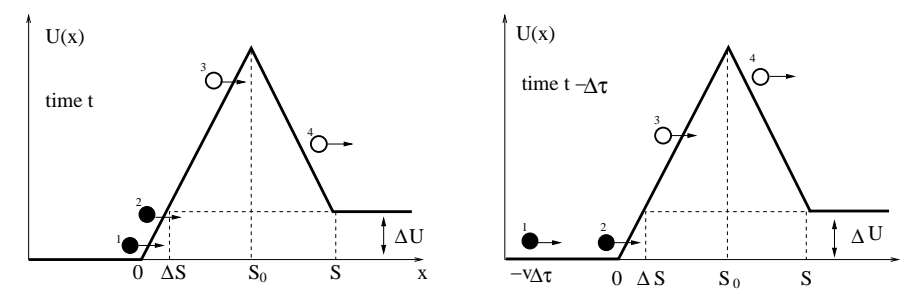

Figure 3: Left figure: a snapshot of four fast $L$-molecules with the same initial velocity $v>v_{a}$. Only molecules located in the interval $0<x<\Delta S$ (molecules 1 and 2) give nonzero contribution to the average force $\left\langle F_{L}\right\rangle$ exerted on the particle by $L$-molecules. Contributions from molecules with $\Delta S<x<S_{0}$ (molecule 3) and $S_{0}<x<S$ (molecule 4) cancel out. Right figure: a snapshot of the same molecules at an earlier time $t-\Delta \tau$, when molecules 1 and 2 were are outside the particle.

which is the minimal speed for $L$-molecules to cross the barrier. The contribution $F_{L}^{(2)}$ corresponds to "fast" molecules with initial velocities $v>v_{a}$.

For molecules with $v<v_{a}$ the barrier is impenetrable, and in order to evaluate $F_{L}^{(1)}$ one can borrow the results of the previous section. Namely, as illustrated in Fig. 2, one can write $F_{L}^{(1)}$ at a given time $t$ in terms of the phase density of molecules at an earlier time $t-\tau_{r}$ as follows

$$
F_{L}^{(1)}(t)=f \int_{0}^{v_{a}} d v \int_{-v \tau_{r}}^{0} d x N\left(x, v, t-\tau_{r}\right) .
$$

Here the residence time $\tau_{r}(v)$ is given by (11), and $f=|d U / d x|$. For the potential-free region $\langle N(x, v, t)\rangle=$ $n f_{M}(v)$, then from (25) and (11) one obtains

$$
\left\langle F_{L}^{(1)}\right\rangle=2 m n \int_{0}^{v_{a}} d v f_{M}(v) v^{2} .
$$

In the limit of impermeable particle, $v_{a} \rightarrow \infty$, the above equation gives the result of the elementary kinetic theory for the pressure force $\langle F\rangle=m n\left\langle v^{2}\right\rangle=n / \beta$.

Consider now the second part $F_{L}^{(2)}$ in Eq.(23), which comes from fast molecules with initial velocities $v>v_{a}$. Such molecules cross the barrier and can occupy both sides of the potential hill. Clearly, the average forces exerted by fast $L$-molecules on the left and right slopes cancel out, except for the molecules near the base of the left slope with coordinates $0<x<\Delta S$, see Fig. 3. Let $\Delta \tau$ be the time for an $L$-molecule with initial velocity $v$ to climb to the altitude $\Delta U$,

$$
\Delta \tau=\frac{m v}{f}\left\{1-\sqrt{1-\frac{2 \Delta U}{m v^{2}}}\right\} \approx \frac{\Delta U}{f v} .
$$

Similar to (25), one can write

$$
F_{L}^{(2)}(t)=f \int_{v_{a}}^{\infty} d v \int_{-v \Delta \tau}^{0} d x N(x, v, t-\Delta \tau)
$$

which gives

$$
\left\langle F_{L}^{(2)}\right\rangle=n \Delta U \int_{v_{a}}^{\infty} d v f_{M}(v) .
$$

The same method can be applied to evaluate two parts of the force

$$
F_{R}=F_{R}^{(1)}+F_{R}^{(2)}
$$


exerted on the particle by $R$-molecules. The only difference is that for molecules coming from the right the potential barrier is lower, $U_{a}-\Delta U$, and so is the activation velocity $\tilde{v}_{a}=\left[2\left(U_{a}-\Delta U\right) / m\right]^{1 / 2}$. In the linear approximation,

$$
\tilde{v}_{a} \approx v_{a}-\Delta v_{a}, \quad \Delta v_{a}=\frac{v_{a} \Delta U}{2 U_{a}},
$$

where $v_{a}$ is the activation velocity for the $L$-molecules given by Eq.(24). Using the same argument as for $L$-molecules, the contribution from slow $R$-molecules with $|v|<\tilde{v}_{a}$, which cannot cross the barrier, can be written as

$$
\left\langle F_{R}^{(1)}\right\rangle=-2 m n \int_{0}^{\tilde{v}_{a}} d v f_{M}(v) v^{2} .
$$

In the linear approximation this takes the form

$$
\left\langle F_{R}^{(1)}\right\rangle=-2 m n \int_{0}^{v_{a}} d v f_{M}(v) v^{2}+2 m n f_{M}\left(v_{a}\right) v_{a}^{2} \Delta v_{a}
$$

or

$$
\left\langle F_{R}^{(1)}\right\rangle=-\left\langle F_{L}^{(1)}\right\rangle+2 n f_{M}\left(v_{a}\right) v_{a} \Delta U
$$

The contribution from fast $R$-molecules with $|v|>\tilde{v}_{a}$ is similar to Eq. (29) for fast $L$-molecules. In fact, in the linear approximation the two expressions coincide,

$$
\left\langle F_{R}^{(2)}\right\rangle=n \Delta U \int_{\tilde{v}_{a}}^{\infty} d v f_{M}(v) \approx n \Delta U \int_{v_{a}}^{\infty} d v f_{M}(v)=\left\langle F_{L}^{(2)}\right\rangle
$$

Combining the above results, the total average fluctuating force can be written as

$$
\langle F\rangle=\left\{2 n f_{M}\left(v_{a}\right) v_{a}+2 n \int_{v_{a}}^{\infty} d v f_{M}(v)\right\} \Delta U
$$

Explicitly this reads

$$
\langle F\rangle=n \phi\left(\frac{v_{a}}{v_{T}}\right) \Delta U
$$

where the dimensionless function $\phi(x)$ is

$$
\phi(x)=\frac{2}{\sqrt{2 \pi}} x \exp \left(-\frac{x^{2}}{2}\right)+\operatorname{erfc}\left(\frac{x}{\sqrt{2}}\right) .
$$

Here the first and second terms originate from slow and fast molecules, respectively. For large $x$ (high barrier/low temperature) the second term is smaller than the first one by the factor of order $1 / x^{2}$ and can be neglected. Then, since $v_{a} / v_{T}=\left(2 \beta U_{a}\right)^{1 / 2}$, one gets

$$
\langle F\rangle=\frac{2}{\sqrt{\pi}} n \sqrt{\beta U_{a}} e^{-\beta U_{a}} \Delta U
$$

This and the expression (7) for $\gamma$ give for the stationary drift velocity $\langle V\rangle=\langle F\rangle / \gamma$ of the motor the following result

$$
\langle V\rangle=\sigma \Delta U, \quad \sigma=\frac{1}{2 \sqrt{2}} \beta \sqrt{\frac{U_{a}}{m}} e^{-\beta U_{a}} .
$$

As expected, the temperature dependence of the mobility $\sigma$ is Arrhenius-like. The square-root dependence of pre-exponential factor on the barrier's height $U_{a}$ is perhaps a less obvious result. The mobility $\sigma$ depends neither on the mass of the motor nor on the concentration of molecules of the thermal bath. 

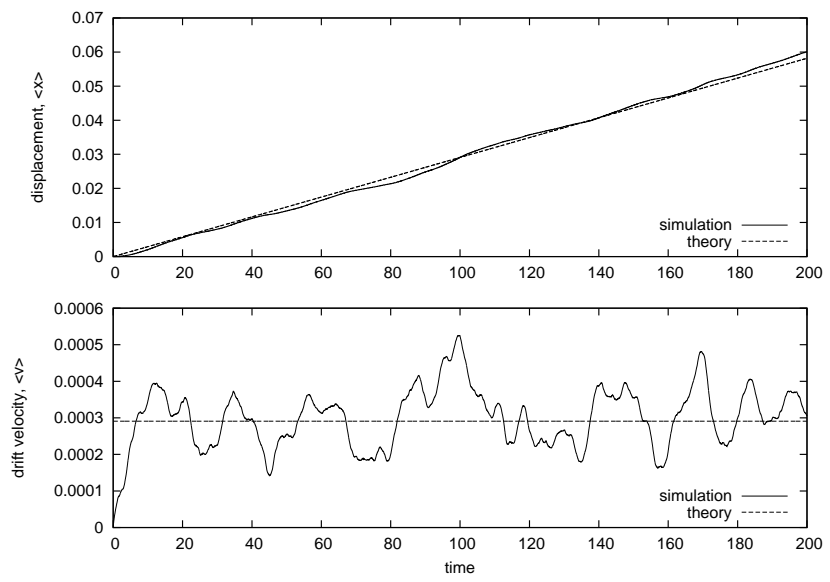

Figure 4: Numerical simulation of the drift characteristics of the motor with $\beta U_{a}=5, \beta \Delta U=0.05$, and mass ratio $m / M=0.1$. Upper figure: average displacement of the motor as a function of time. Lower figure: average drift velocity. Solid lines correspond to numerical experiment data, averaged over $1.8 \cdot 10^{7}$ trajectories. Dashed lines represent theoretical predictions given by Eq.40). Units of velocity, time, and displacement are, respectively, $v_{0}=v_{T}, \tau_{0}=\tau_{r}\left(v_{T}\right) / 2$, and $x_{0}=v_{0} \tau_{0}$.

\section{Discussion}

The autonomous mesoscopic motor described in this paper is based on a permeable Brownian particle which propels due to asymmetric transfer characteristics, and in this sense may be called a Brownian diode. The main result (39) for the motor's mobility $\sigma$ is obtained under asymptotic conditions of a high barrier and small potential drop (4). To verify this result, we performed a one-dimensional numerical simulation similar to standard molecular dynamics with the velocity Verlet algorithm. Instead of periodic boundary conditions that poorly fit the problem, a very large thermal bath with a Maxwellian velocity distribution (11) and a constant density of molecules was generated in the beginning of each simulation run. The size of the bath was of order $c v_{T} t_{s}$, where $t_{s}$ is the duration of one simulation run, and $c$ is a numerical parameter of order $10^{2}$. As far as the Brownian particle dynamics is concerned, the difference between a bath of such size and the infinite one is that the particle immersed in the former may feel a deficit of hot molecules with velocities higher than $c v_{T}$. For $c \gtrsim 10^{2}$, it was found empirically that results do not depend on $c$, and thus the difference with the infinite bath is expected to be negligible. The assumption of noninteracting bath molecules strongly reduces the amount of computations. It also allows some optimization by eliminating molecules with initial velocity directions away from the particle and also those which are so slow and generated so far from the particle that cannot come to its proximity during the time of simulation. The ensemble average of the particle's coordinate and velocity as functions of time is taken by performing multiple runs, resetting the bath molecules with new initial conditions and averaging over the simulation runs.

Simulation shows a good agreement with the prediction (39) for small $\Delta U$ and when $\beta U_{a} \gtrsim 5$, see Fig.4. The agreement gets better when one takes into account the contribution of fast molecules given by the second term in Eq.(37). Since $\operatorname{erfc}(x) \approx e^{-x^{2}} / \sqrt{\pi} x$ for large $x$, the corrected expression for the mobility takes the form

$$
\sigma=\frac{1}{2 \sqrt{2}} \beta \sqrt{\frac{U_{a}}{m}}\left(1+\frac{1}{2 \beta U_{a}}\right) e^{-\beta U_{a}} .
$$

For lower barrier heights, $\beta U_{a}<5$, the simulation shows that Eqs.(39) and (40) both noticeably underestimate the mobility, see Fig. 5. The discrepancy comes from the approximation of an infinitely high barrier, which we have used to calculate the friction coefficient $\gamma$, Eq. (22). Clearly, this overestimates $\gamma$ and underestimates the drift velocity $\langle V\rangle=\langle F\rangle / \gamma$. An analytical evaluation of $\gamma$ for the case of a finite barrier is feasible but somewhat cumbersome. One can instead find $\gamma$ from a numerical experiment measuring 

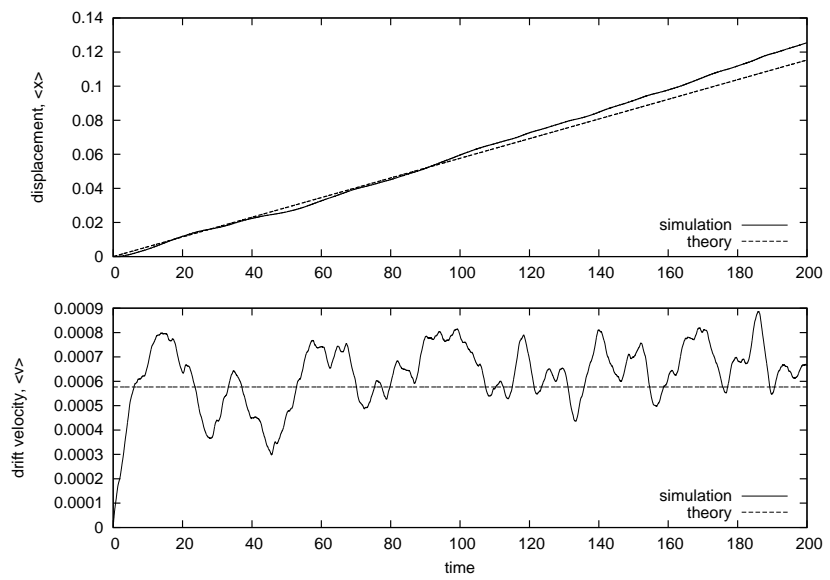

Figure 5: Same as Fig. 4, but for a lower potential barrier $\beta U_{a}=4$. For a lower barrier the disagreement of experimental and theoretical results becomes more significant.

velocity relaxation $\langle V(t)\rangle=V_{0} \exp (-\gamma t)$. When one uses $\gamma$ found in this way, and $\langle F\rangle$ given by (36), a good agreement with the numerical experiment may be restored. (This method is not very effective for the mass ratio $m / M \gtrsim 0.1$, when relaxation of the Brownian particle's velocity is distinctly non-exponential.)

In our calculations, we assumed that the two slopes of the potential hill are equally steep. No qualitatively new feature appears if they are not (provided the potential is continuous). As shown above, the stationary mobility does not depend on $U^{\prime}(x)$. Respectively, for an asymmetric barrier with no potential drop, the drift may be only transient and vanishes in the long-time limit. This is confirmed by numerical simulation for regimes within and beyond the linear response. At this point, however, it perhaps should be recalled that in this study we ignored the particle's internal degrees of freedom. Taking them into account, one may expect that the model would reveal new features characteristic for granular Brownian motors, interacting with bath molecules via inelastic collisions 17.

\section{References}

[1] C. Doering, B. Ermentrout, and G. Oster, Biophys. J. 69, 2256 (1995).

[2] D. Astumian, Science, 276, 917 (1997), Phys. Chem. Chem. Phys. 9, 5067 (2007).

[3] P. Reimann, Phys. Rep. 361, 57 (2002).

[4] R. Golestanian, T. B. Liverpool, and A. Ajdari, Phys. Rev. Lett. 94, 220801 (2005).

[5] G. Rückner and R. Kapral, Phys. Rev. Lett 98, 150603 (2007).

[6] B. R. Saunders, N. Laaiam, E. Daly, S. Teow, X. Hu, and R. Stepto, Adv. Colloid Interface Sci 147-148, 251 (2009).

[7] J. de Gier, Chem. Phys. Lipids 64, 187 (1993).

[8] A. A. Gurtovenko and I. Vattulainen, J. Phys. Chem. B 112, 4629 (2008).

[9] A. Gomez-Marin and J. M. Sancho, EPL 86, 40002 (2009).

[10] P. K. Ghosh, A. Y. Smirnov, and F. Nori, J. Chem. Phys. 131, 035102 (2009)

[11] J. K. Lanyi and A. Pohorille, Trends Biotechnol. 19, 140 (2001).

[12] C. Van den Broeck and K. Lindenberg, Phys. Rev. E 86, 041144 (2012).

[13] G. W. Parker, Am. J. Phys. 70, 502 (2002).

[14] A. V. Plyukhin and J. Schofield, Phys. Rev. E 68, 041107 (2003).

[15] For the model with instantaneous binary collisions it is easier to evaluate the friction coefficient from the Fokker-Planck equation, see e.g. N.G. van Kampen, Stochastic processes in physics and chemistry, North-Holland, Amsterdam, 1981.

[16] R. M. Mazo, Brownian Motion, Oxford University Press, 2002.

[17] G. Constantini, U. M. B. Marconi, A. Puglisi, Phys. Rev. E 75061124 (2007); B. Cleuren and C. Van den Broeck, Europhys. Lett. 77, 50003 (2007); B. Cleuren and R. Eichhorn, J. Stat. Mech. P10011 (2008); J. Talbot, R. D. Wildman, and P. Viot, Phys. Rev. Lett. 107, 138001 (2011). 